PROCEEDINGS OF THE

AMERICAN MATHEMATICAL SOCIETY

Volume 129, Number 10, Pages 3017-3023

S 0002-9939(01)06142-1

Article electronically published on April 24, 2001

\title{
AN EXAMPLE OF AN ASYMPTOTICALLY HILBERTIAN SPACE WHICH FAILS THE APPROXIMATION PROPERTY
}

\author{
P. G. CASAZZA, C. L. GARCÍA, AND W. B. JOHNSON
}

(Communicated by N. Tomczak-Jaegermann)

\begin{abstract}
Following Davie's example of a Banach space failing the approximation property (1973), we show how to construct a Banach space $E$ which is asymptotically Hilbertian and fails the approximation property. Moreover, the space $E$ is shown to be a subspace of a space with an unconditional basis which is "almost" a weak Hilbert space and which can be written as the direct sum of two subspaces all of whose subspaces have the approximation property.
\end{abstract}

\section{INTRODUCTION}

This paper is concerned with the relationship between the approximation property and notions about Banach spaces which are in some sense close to Hilbert space, namely, the notions of asymptotically Hilbertian space and of weak Hilbert space.

The spaces we discuss are of the form $Z=\left(\sum_{n=0}^{\infty} \ell_{p_{n}}^{k_{n}}\right)_{2}$ with $p_{n} \downarrow 2$ and $k_{n} \uparrow \infty$. It is easy to check that any space of this form is an asymptotically Hilbertian space (see below for definitions). For particular sequences $\left(p_{n}\right)$ and $\left(k_{n}\right)$ we show that such a $Z$ has a subspace $E$ failing the approximation property. Moreover, we can choose a subsequence of $\left(p_{n}\right)$, such that if $N_{1}=\left\{j \mid p_{n_{2 k+1}} \leq j<p_{n_{2(k+1)}}, k \geq 0\right\}$ and $N_{2}=\mathbb{N}-N_{1}$, then for $Z_{i}=\left(\sum_{j \in N_{i}} \ell_{p_{j}}^{k_{j}}\right)_{2}, i=1,2$, we have that $Z=Z_{1} \oplus Z_{2}$ and that all subspaces of $Z_{1}$ and of $Z_{2}$ have the approximation property ([]).

The construction of $E$ provides quantitative estimates which show that $Z$, and hence also $E$, is surprisingly close to being a weak Hilbert space (note that weak Hilbert spaces enjoy the approximation property $[\mathrm{P}]$ ).

First we recall the notion of asymptotically Hilbertian space. Given integers $n \geq 0, m \geq 1$ and a constant $K$, say that $X$ satisfies $H(n, m, K)$ provided there is an $n$-codimensional subspace $X_{m}$ of $X$ so that every $m$-dimensional subspace of $X_{m}$ is $K$-isomorphic to $\ell_{2}^{m}$. A Banach space $X$ is said to be asymptotically Hilbertian provided there is a constant $K$ so that for every $m$ there exists $n$ so that $X$ satisfies $H(n, m, K)$. Since here we are interested in good estimates, we

Received by the editors March 1, 2000.

2000 Mathematics Subject Classification. Primary 46B20, 46B07, 46B28; Secondary 46B99.

Key words and phrases. Banach spaces, weak Hilbert spaces, asymptotically Hilbertian, approximation property.

The first author was supported by NSF grant DMS-970618.

The second and third authors were supported in part by NSF grants DMS-9623260, DMS9900185, and by the Texas Advanced Research Program under Grant No. 010366-163. 
denote by $H_{X}(m, K)$ the smallest $n$ for which $X$ has $H(n, m, K)$. Thus if $X$ is $K$-isomorphic to a Hilbert space, then $H_{X}(m, K)=0$ for all $m$. The growth rate of $H_{X}(m, K)$ for a fixed $K$ as $m \rightarrow \infty$ is one measurement of the closeness of $X$ to a Hilbert space.

A Banach space is called a weak Hilbert space provided that there are positive constants $\delta$ and $K$ so that for every $n$, every $n$-dimensional subspace of $X$ contains a further subspace $E$ of dimension at least $\delta n$ so that $E$ is $K$-isomorphic to a Hilbert space and $E$ is $K$-complemented in $X$ (that is, there is a projection having norm at most $K$ from $X$ onto $E$ ).

The definition of the property $H(n, m, K)$ was made in [J], although the nomenclature "asymptotically Hilbertian" was coined by Pisier $[\mathrm{P}]$. Weak Hilbert spaces were introduced by Pisier $[\mathrm{P}$, who gave many equivalences to the property of being weak Hilbert; we chose the one most relevant for this paper as the definition of weak Hilbert.

Relations between the weak Hilbert property and the asymptotically Hilbertian property are given in [J] and [P. First, a weak Hilbert space must be asymptotically Hilbertian ([P Section 4]). It seems likely that if $X$ is weak Hilbert, then for some $K, H_{X}(m, K) \leq K m$, but in fact no reasonable estimates are known for $H_{X}(m, K)$ when $X$ is a weak Hilbert space. It is known (see [CJT], [NT-J]) that if $X$ is a weak Hilbert space which has an unconditional basis and there is a $K$ so that $H_{X}(m, K)$ is dominated by $f(m)$ for some iterate $f$ of exp, then for any iterate $g$ of log there is another constant $K^{\prime}$ so that $H_{X}\left(m, K^{\prime}\right) \leq K^{\prime} g(m)$. In the other direction, it follows from [J] that if for some $K$ the sequence $H_{X}(m, K)$ grows sufficiently slowly as $m \rightarrow \infty$ (say, like $\log \log m$ ), then $X$ is a weak Hilbert space. In this paper we are interested in examples of spaces which are of type 2. We refer to Chapter 11 of [DJT] or Section 1.4 of [T-J] for the definitions and basic theory of type $p$ and cotype $p$ as well and the type $p$ and cotype $p$ constants $T_{p}(X), C_{p}(X)$ of a Banach space $X$. Relevant for us is that if $X$ is a type 2 space and $E$ is a subspace of $X$ which is $K$-isomorphic to a Hilbert space, then, by Maurey's extension theorem, $E$ is $T_{2}(X) K$-complemented in $X$ (DJT, Corollary 12.24]). Thus it is clear that if $X$ is of type 2 and for some $K, H_{X}(m, K) \leq K m$, then $X$ is weak Hilbert. Here we should mention that by [FLM], polynomial growth of $H_{X}(m, K)($ as $m \rightarrow \infty)$ implies linear growth of $H_{X}\left(m, K^{\prime}\right)$ for some $K^{\prime}$.

Our main interest here is the linkage among the weak Hilbert property, the asymptotically Hilbertian property, and the approximation property. The arguments in J] show that if $X$ has type 2 and for some $K, H_{X}(m, K) \leq K \log m$ for infinitely many $m$, then all subspaces of $X$ (even all subspaces of every quotient of $X$ ) have the approximation property. In $[\mathrm{P}$ it is shown that all weak Hilbert spaces have the approximation property. Thus if $X$ is of type 2 and for some $K, H_{X}(m, K) \leq K m$ for all $m$, then all subspaces of every quotient of $X$ have the approximation property. It is easy to build examples of a type 2 space $X$ for which there is a constant $K$ so that for any iterate $f$ of the log function, $H_{X}(m, K) \leq K f(m)$ for infinitely many $m$ and yet $X$ is not a weak Hilbert space. Now such a space $X$ is in some sense close to Hilbert space and, in particular, every subspace of $X$ has the approximation property. In this paper we show that there are two such spaces, call them $Z_{1}$ and $Z_{2}$, so that $Z:=Z_{1} \oplus Z_{2}$ has a subspace which fails the approximation property. Moreover, $Z$ has an unconditional basis $\left(Z=\sum_{n=0}^{\infty} \ell_{p_{n}}^{k_{n}}\right.$ for appropriate $p_{n} \downarrow 2$ and $\left.k_{n} \uparrow \infty\right)$ and is nearly a weak Hilbert space in the sense that for some $K$, the growth rate of $H_{Z}(m, K)$ as $m \rightarrow \infty$ is 
close to being polynomial in $m\left(H_{Z}(m, K) \leq m^{\log \log m}\right.$ is what we get; recall that polynomial growth of $H_{X}(m, K)$ gives linear growth of $H_{X}\left(m, K^{\prime}\right)$ for some $\left.K^{\prime}\right)$.

\section{The EXAMPLe}

We closely follow A. M. Davie's construction of a Banach space failing the approximation property [D. Davie constructed for $p>2$ a subspace of $\ell_{p}=\left(\sum \ell_{p}^{k_{n}}\right)_{p}$ which fails the approximation property. He could as well have used $\left(\sum \ell_{p}^{k_{n}}\right)_{r}$ for any $1 \leq r \leq \infty$. Here we use instead $Z:=\left(\sum_{n=0}^{\infty} \ell_{p_{n}}^{k_{n}}\right)_{2}$ where $p_{n} \downarrow 2$ appropriately and $k_{n}$ as in [D]. Basically we compute how fast $p_{n}$ can go to 2 so that Davie's argument yields a subspace of $Z$ which fails the a.p. The obvious condition is that $k_{n}^{1 / 2-1 / p_{n}}$ cannot be bounded, for if $k_{n}^{1 / 2-1 / p_{n}}$ is bounded, then $\left(\sum_{n=0}^{\infty} \ell_{p_{n}}^{k_{n}}\right)_{2}$ is isomorphic to $\ell_{2}$.

For any integer $n \geq 0$ consider an Abelian group $G_{n}$ of order $k_{n}=3 \cdot 2^{n}$, and let $\sigma_{1}^{n}, \ldots, \sigma_{2^{n}}^{n}, \tau_{1}^{n}, \ldots, \tau_{2^{n+1}}^{n}$ be the characters of $G_{n}$. Lemma $(b)$ in $\mathrm{D}$ ] shows that this enumeration of the characters of $G_{n}$ can be chosen so that there exists an absolute constant $A>0$ such that for all $g \in G_{n}$,

$$
\left|2 \sum_{j=1}^{2^{n}} \sigma_{j}^{n}(g)-\sum_{j=1}^{2^{n+1}} \tau_{j}^{n}(g)\right| \leq A(n+1)^{1 / 2} 2^{n / 2} .
$$

Let $G$ be the disjoint union of the sets $G_{n}$ and for each $n \geq 0$ and $1 \leq j \leq 2^{n}$ define $e_{j}^{n}: G \rightarrow \mathbb{C}$ via

$$
e_{j}^{n}(g)=\left\{\begin{array}{cc}
\tau_{j}^{n-1}(g), & \text { if } g \in G_{n-1}, n \geq 1, \\
\varepsilon_{j}^{n} \sigma_{j}^{n}(g), & \text { if } g \in G_{n}, \\
0, & \text { otherwise }
\end{array}\right.
$$

where $\varepsilon_{j}^{n}= \pm 1$ is a choice of signs for which the inequality (2.5) below is satisfied.

To define $E$ let, as above, $k_{n}=3 \cdot 2^{n}$ and let $\left(p_{n}\right)_{n=0}^{\infty}, 2<p_{n} \leq 3$, be a decreasing sequence converging to 2 . The appropriate rate of decrease of the sequence $\left(p_{n}\right)_{n=0}^{\infty}$ will be chosen later.

Let $Z=\left(\sum_{n=0}^{\infty} \ell_{p_{n}}^{k_{n}}\right)_{2}$ which in our setting is

$$
Z=\left\{f: G \rightarrow \mathbb{C} \mid \sum_{n=0}^{\infty}\left(\sum_{g \in G_{n}}|f(g)|^{p_{n}}\right)^{2 / p_{n}}<\infty\right\} .
$$

Define $E$ to be the closed linear span in $Z$ of $\left\{e_{j}^{n} \mid n \geq 0,1 \leq j \leq 2^{n}\right\}$. To show that $E$ fails the approximation property one proceeds as follows:

For $n \geq 0$ and $1 \leq j \leq 2^{n}$ define $\alpha_{j}^{n} \in E^{*}$ by

$$
\alpha_{j}^{n}(f)=3^{-1} 2^{-n} \sum_{g \in G_{n}} \varepsilon_{j}^{n} \sigma_{j}^{n}\left(g^{-1}\right) f(g) .
$$

When $n \geq 1$ the expression above equals

$$
\alpha_{j}^{n}(f)=3^{-1} 2^{1-n} \sum_{g \in G_{n-1}} \tau_{j}^{n-1}\left(g^{-1}\right) f(g) .
$$

This follows from the fact that $\alpha_{j}^{n}\left(e_{i}^{k}\right)=\delta_{i j} \cdot \delta_{k n}$ (because of the orthogonality of the characters of a group) and then a linearity and continuity argument shows that (2.2) and (2.3) agree on $E$. 
Now let $B(E)$ be the space of bounded, linear operators on $E$, and for each $n \geq 0$ define $\beta^{n}$ in the dual space $B(E)^{*}$ as

$$
\beta^{n}(T)=2^{-n} \sum_{n=1}^{2^{n}} \alpha_{j}^{n}\left(T\left(e_{j}^{n}\right)\right), \quad T \in B(E) .
$$

Using (2.2) we can rewrite $\beta^{n}$ as

$$
\beta^{n}(T)=3^{-1} 4^{-n} \sum_{g \in G_{n}} T\left(\sum_{j=1}^{2^{n}} \varepsilon_{j}^{n} \sigma_{j}^{n}\left(g^{-1}\right) e_{j}^{n}\right)(g),
$$

and from (2.3) we get

$$
\beta^{n+1}(T)=6^{-1} 4^{-n} \sum_{g \in G_{n}} T\left(\sum_{j=1}^{2^{n+1}} \tau_{j}^{n}\left(g^{-1}\right) e_{j}^{n+1}\right)(g) .
$$

Hence,

$$
\beta^{n+1}(T)-\beta^{n}(T)=3^{-1} 2^{-n} \sum_{g \in G_{n}} T\left(\Phi_{g}^{n}\right)(g)
$$

where

$$
\Phi_{g}^{n}=2^{-n-1} \sum_{j=1}^{2^{n+1}} \tau_{j}^{n}\left(g^{-1}\right) e_{j}^{n+1}-2^{-n} \sum_{j=1}^{2^{n}} \varepsilon_{j}^{n} \sigma_{j}^{n}\left(g^{-1}\right) e_{j}^{n}, \quad g \in G_{n} .
$$

Note that $\Phi_{g}^{n} \in E$ for every $g \in G_{n}$ and $n \geq 1$. Now we estimate the right-hand side of (2.4). If $n \geq 1$ and $g \in G_{n}$, then

$$
3^{-1} 2^{-n} \sum_{g \in G_{n}}\left|T\left(\Phi_{g}^{n}\right)(g)\right| \leq \sup _{g \in G_{n}}\left\{\left\|T\left(\Phi_{g}^{n}\right)\right\|_{\infty}\right\} \leq \sup _{g \in G_{n}}\left\{\left\|T\left(\Phi_{g}^{n}\right)\right\|_{Z}\right\} .
$$

Therefore,

$$
\left|\beta^{n+1}(T)-\beta^{n}(T)\right| \leq \sup _{g \in G_{n}}\left\{\left\|T\left(\Phi_{g}^{n}\right)\right\|_{Z}\right\} \text { for every } T \in B(E) .
$$

Note that from (2.1) we have that $\left|\Phi_{g}^{n}(h)\right| \leq A(n+1)^{1 / 2} 2^{-n / 2}$ for $g, h \in G_{n}$. By applying lemma (a) in $\left[\mathrm{D}\right.$, the signs $\varepsilon_{j}^{n}, 1 \leq j \leq 2^{n}$, can be chosen so that

$$
\left|\Phi_{g}^{n}(h)\right| \leq A_{2}(n+1)^{1 / 2} 2^{-n / 2} \quad \text { for } g \in G_{n}, h \in G_{n-1} \quad(n \geq 1)
$$

where $A_{2}$ is some absolute constant. An algebraic argument shows that a similar estimate can be obtained for $g \in G_{n}$ and $h \in G_{n+1}$. In brief, we have that there is an absolute constant, say $A$, such that

$$
\left|\Phi_{g}^{n}(h)\right| \leq A(n+1)^{1 / 2} 2^{-n / 2} \quad \text { for } g \in G_{n} \text { and } h \in G_{n-1} \sqcup G_{n} \sqcup G_{n+1} .
$$

Now, if $n \geq 1$ and $g \in G_{n}$, then

$$
\begin{aligned}
\left\|\Phi_{g}^{n}\right\|_{Z}^{2} & =\sum_{j=n-1}^{n+1}\left(\sum_{h \in G_{j}}\left|\Phi_{g}^{n}(h)\right|^{p_{j}}\right)^{2 / p_{j}} \\
& \leq A^{2}(n+1) 2^{-n}\left(\left(3 \cdot 2^{n-1}\right)^{2 / p_{n-1}}+\left(3 \cdot 2^{n}\right)^{2 / p_{n}}+\left(3 \cdot 2^{n+1}\right)^{2 / p_{n+1}}\right) \\
& \leq 3 A^{2}(n+1) 2^{-n}\left(2^{2(n-1) / p_{n-1}}+2^{2 n / p_{n}}+2^{2(n+1) / p_{n+1}}\right) \\
& \leq 18 A^{2}(n+1) 2^{2 n\left(1 / p_{n+1}-1 / 2\right)} .
\end{aligned}
$$


Thus,

$$
\left\|\Phi_{g}^{n}\right\|_{Z} \leq 3 \sqrt{2} A(n+1)^{1 / 2} 2^{n\left(1 / p_{n+1}-1 / 2\right)} .
$$

Consider the set

$$
\mathcal{C}=\left\{e_{1}^{0}\right\} \cup\left\{(n+1)^{2} \Phi_{g}^{n} \mid g \in G_{n}, n \geq 1\right\} .
$$

The estimate in (2.7) clearly shows that when

$$
(n+1)^{5 / 2} 2^{n\left(1 / p_{n+1}-1 / 2\right)} \rightarrow 0
$$

the set $\mathcal{C}$ becomes a relatively compact subset of $E$. Obviously there are many choices for $\left(p_{n}\right)_{n=0}^{\infty}, p_{n} \downarrow 2$, that satisfy (2.8); in particular, $1 / p_{n}=1 / 2-1 /(n+1)^{\alpha}$ for any $\alpha<1$ gives a sequence satisfying (2.8). When

$$
n\left(1 / p_{n+1}-1 / 2\right)=-3 \log _{2}(n+1),
$$

the sequence $\left(p_{n}\right)$ is the one with the slowest (up to a constant) possible rate of decrease for this construction. This makes the space $Z$ "almost" a weak Hilbert space in the sense that for some $K, H_{Z}(m, K) \leq m^{\log \log m}$ for large $m$. Indeed, consider $F$, a subspace of $\left(\sum_{j=n+1}^{\infty} \ell_{p_{j}}^{k_{j}}\right)_{2}$ of dimension $m=m\left(k_{1}+\cdots+k_{n}\right)$, where $m$ is the largest integer such that $0<1 / 2-1 / p_{n+1}<1 / \log _{2}(m)$. Then, $d\left(F, \ell_{2}^{m}\right) \leq T_{2}(F) C_{2}(F)$. The type 2 constant of $F$ is bounded by an absolute constant independent of $m$, say $c_{1}$. The cotype 2 constant of $F$ can be estimated, using Tomczak's lemma, by the cotype 2 constant $C_{2, m}(\cdot)$ on $m$ vectors (see Section 5.25 in [T-J]]):

$$
\begin{aligned}
C_{2}(F) \leq \sqrt{2} C_{2, m}(F) & \leq \sqrt{2} C_{p_{n+1}}(F) m^{1 / 2-1 / p_{n+1}} \\
& \leq \sqrt{2} c_{2} m^{1 / 2-1 / p_{n+1}} \\
& \leq 2 \sqrt{2} c_{2} \quad \text { (by the choice of } m \text { ). }
\end{aligned}
$$

Hence, for $K:=2 \sqrt{2} c_{1} c_{2}$ we obtain that $d\left(F, \ell_{2}^{m}\right) \leq K$.

Finally, to show that $E$ fails the approximation property the argument in $[\mathrm{D}$ ] finishes as follows: for every $T \in B(E)$,

$$
\left|\beta^{n+1}(T)-\beta^{n}(T)\right| \leq \sup _{g \in G_{n}}\left\{\left\|T\left(\Phi_{g}^{n}\right)\right\|_{Z}\right\} \leq(n+1)^{-2} \sup _{x \in \mathcal{C}}\|T x\|_{Z} .
$$

Also,

$$
\left|\beta^{0}(T)\right| \leq\left\|T e_{0}^{1}\right\| \leq \sup _{x \in \mathcal{C}}\|T x\|_{Z}
$$

Hence $\beta(T)=\lim _{n \rightarrow \infty} \beta^{n}(T)$ exists for all $T \in B(E)$ and satisfies

$$
|\beta(T)| \leq 3 \sup _{x \in \mathcal{C}}\|T x\|_{Z} .
$$

In particular, when $\mathcal{C}$ is compact, $\beta$ is a continuous linear functional on $B(E)$ when $B(E)$ is given the topology of uniform convergence on compact sets.

If $I_{E}$ is the identity map on $E$, it follows from the definition of $\beta^{n}$ that $\beta^{n}\left(I_{E}\right)=1$ for all $n$, so $\beta\left(I_{E}\right)=1$. On the other hand it is easy to see that $\beta$ vanishes on the set of finite rank operators on $E$, thus $E$ cannot have the approximation property. 
Remark 2.1. For $\left(p_{n}, k_{n}\right)_{n=0}^{\infty}$ as above, we obtained an asymptotically Hilbertian space $Z$ which has a subspace failing the approximation property. The space $Z$ can be decomposed as the direct sum of two subspaces, say $Z_{1}$ and $Z_{2}$, all of whose subspaces have the approximation property. Indeed, as in example 1.g.7 in [LT, it is enough to construct a subsequence $\left(p_{n_{j}}\right)$ of $\left(p_{n}\right)$ as follows: set $p_{n_{1}}=p_{0}$ and $k_{n_{1}}=k_{0}$. Having chosen $p_{n_{1}} \cdots p_{n_{j}}$ (and their respective $k_{n_{1}} \cdots k_{n_{j}}$ ), choose $p_{n_{j+1}}$ such that if $F \subset \ell_{p}\left(2<p<p_{n_{j+1}}\right)$ has dimension $m \leq 2 \cdot 5^{\sum_{i=1}^{j} k_{n_{i}}}$, then $m^{1 / 2-1 / p_{n_{j+1}}} \leq 2$ (in particular $d\left(F, \ell_{2}^{m}\right) \leq 2$ ). Now set $N_{1}=\left\{j \mid p_{n_{2 k+1}} \leq j<\right.$ $\left.p_{n_{2(k+1)}}, k \geq 0\right\}$ and $N_{2}=\mathbb{N}-N_{1}$. Let $Z_{i}=\left(\sum_{j \in N_{i}} \ell_{p_{j}}^{k_{j}}\right)_{2}, i=1,2$.

Our example is the best possible in light of current theory and the current wisdom in the field. First, it follows from the arguments in [J] that the spaces $Z_{1}, Z_{2}$ have the property that every subspace of every quotient space of $Z_{i}, i=1,2$, has a decomposition of the form $Z_{i}=\left(\sum_{k=1}^{\infty} E_{k}\right)_{\ell_{2}}$, where $\operatorname{dim} E_{k}<\infty$ for each $k=1,2,3, \ldots$ Also, the argument of Szarek $[\mathbf{S}]$ shows that the spaces $Z_{i}$ have subspaces without bases. One might try to refine this example to produce $Z_{i}$ 's for which every subspace has a basis. However, this may not be possible since it is an open question of whether Banach spaces for which every subspace has a basis must be weak Hilbert. Since the direct sum of weak Hilbert spaces is weak Hilbert, and every subspace of a weak Hilbert space has the approximation property, a positive answer to this question would show that our construction cannot be improved to produce $Z_{i}$ 's for which every subspace has a basis. It was shown by Maurey and Pisier (see $[\underline{\mathrm{M}}]$ ) that every separable weak Hilbert space $X$ has a finite-dimensional decomposition. That is, there is a sequence of finite-dimensional subspaces $E_{i}$ of $X$ so that for every $x \in X$ there is a unique sequence $x_{i} \in E_{i}$ so that $x=\sum_{i} x_{i}$. However, it is an open question of whether a separable weak Hilbert space must have a basis.

\section{REFERENCES}

[CJT] P. G. Casazza, W. B. Johnson, L. Tzafriri, On Tsirelson's space, Israel J. Math. 47 (1984), 81-98. MR 85m:46013

[D] A. M. Davie, The approximation problem for Banach spaces, Bull. London Math. Soc. 5 (1973), 261-266. MR 49:3499

[DJT] J. Diestel, H. Jarchow, A. Tonge, Absolutely summing operators, Cambridge University Press, Cambridge, 1995. MR 96i:46001

[FLM] T. Figiel, J. Lindenstrauss, V.D. Milman, The dimension of almost spherical sections of convex bodies, Acta Math. 139 (1977), 53-94. MR 56:3618

[J] W. B. Johnson, Banach spaces all whose subspaces have the approximation property, Special Topics of Applied Mathematics, North-Holland (1980), 15-26 (also in Séminaire d'Analyse Fonctionelle 79/80, Ecole Polytechnique. Palaiseau. Exposé No. 16). MR 81m:46032, MR 82d:46024

[LT] J. Lindenstrauss, L. Tzafriri, Classical Banach spaces, Vol. II, Springer-Verlag, New York, 1979. MR 81c:46001

[M] V. Mascioni, On Banach spaces isomorphic to their duals, Houston J. Math. 19 (1993), 27-38. MR 94b:46012

[NT-J] N. J. Nielsen, N. Tomczak-Jaegermann, Banach lattices with property (H) and weak Hilbert spaces, Illinois J. Math. 36 (1992), 345-371. MR 93i:46037

[P] G. Pisier, Weak Hilbert spaces, Proc. London Math. Soc. 56 (1988), 547-579. MR 89d:46022

[S] S. Szarek, A Banach space without a basis which has the bounded approximation property, Acta Math. 159 (1987), 81-98. MR 88f:46029 
[T-J] N. Tomczak-Jaegermann, Banach-Mazur distances and finite-dimensional operator ideals, Pitman Monographs and Surveys in Pure and Applied Mathematics 38, Longman (1989). MR 90k:46039

Department of Mathematics, University of Missouri-Columbia, Columbia, Missouri 65211

E-mail address: pete@math.missouri.edu

Department of Mathematics, Texas A\&M University, College Station, Texas 77843 3368

E-mail address: clgarcia@math.tamu.edu

Department of Mathematics, Texas A\&M University, College Station, Texas 77843 3368

E-mail address: johnson@math.tamu.edu 